Pacific Journal of Mathematics

DOM WALLS AND RES KERN 


\section{RANDOM WALKS AND RIESZ KERNELS}

\section{J. A. Williamson}

It is the purpose of this paper to study the behavior for large $|x-y|$ of the Green Function, $G(x, y)$, of a random walk, $\left\{S_{n}, n \in N\right\}$, having increments belonging to the domain of attraction of a $d$-dimensional stable law with characteristic exponent $\alpha, 0<\alpha<\min (d, 2)$. The main results are concerned with the problem of finding conditions under which $G(0, x)$ is asymptotic to $|x|^{d-\alpha} L(|x|)$ where $L$ is a function of slow growth. The results, including those found in the discussion of the discrete potential theory for such random walks, are for the most part discrete analogs of theorems for transient stable processes.

Throughout this paper the notation, definitions, and conventions of [16] will be used. The position of the random walk at time $n$ will be denoted by $S_{n}$ with $S_{0} \equiv 0$. The independent identically distributed random variables, $S_{k}-S_{k-1}$, will be denoted by $X_{k} . P$ will refer to the measure on the underlying probability space.

$$
\begin{aligned}
\varphi(t) & =E\left(e^{i t \cdot x_{1}}\right)=\int e^{i t \cdot X_{1}} P\{d \omega\} . \\
P_{n}(x, y) & =P_{n}(0, y-x)=P\left\{S_{n}=y-x\right\}, n=1,2, \cdots . \\
P(x, y) & =P_{1}(x, y) \text { and } \delta(x, y)=P_{0}(x, y) . \\
G(x, y) & =\sum_{n=0}^{\infty} P_{n}(x, y) .
\end{aligned}
$$

It follows either from Lemma 2-B or from T8.2 of [16] that for all random walks considered in these pages, $G(x, y)<\infty$ for all $x$ and $y$ in the state space.

The state space, $R$ will always be $\left\{x: x=\left(x_{1}, x_{2}, \cdots x_{d}\right), x_{i}\right.$ is an integer for each $i$. $d$-dimensional random walks are always assumed to be genuinely $d$-dimensional as defined in D7.4 of [16]. Definitions of aperiodic and strongly aperiodic are also found in [16] in D2.2 and D5.1. The set $\left\{t \in E^{d}: t=\left(t_{1}, t_{2}, \cdots t_{d}\right),\left|t_{i}\right| \leqq \pi\right.$ for each $\left.i\right\}$ will be denoted by $C$ and the integration with respect to $t$ of expressions involving $\varphi(t)$ will be over $C$ unless otherwise indicated.

The main result takes the form:

THEOREM. Let $\left\{S_{n}, n \in N\right\}$ be an aperiodic d-dimensional random walk having increments $X_{1}, X_{2}, X_{3}, \cdots$ belonging to the domain of attraction of a nondegenerate d-dimensional stable law with density $g_{\alpha}(x)$ and with characteristic exponent $\alpha, d / 2<\alpha<\min (d, 2)$. If 
$E\left(X_{1}\right)=0$ where it exists, then

$$
\begin{aligned}
& \lim _{|x| \rightarrow \infty}|x|^{d} P(|x|) G(0, x)=\alpha \int_{0}^{\infty} g_{\alpha}\left(v u_{x}\right) v^{d-1-\alpha} d v \\
& \text { uniformly in } u_{x}=x /|x| \text { where } P(y)=\boldsymbol{P}\left\{\left|X_{1}\right|>y\right\} .
\end{aligned}
$$

If $\alpha>1$ or, in the case $d=1$, if $|\beta|<1$ ( $\beta$ defined in (1.5)), then (1.1) can be replaced by the slightly stronger

$$
\lim _{|x| \rightarrow \infty} \frac{|x|^{d} P(|x|) G(0, x)}{\alpha \int_{0}^{\infty} g_{\alpha}\left(v u_{x}\right) v^{d-1-\alpha} d v}=1 \text { uniformly in } u_{x} .
$$

Under the assumptions of this theorem with $d=1$, (1.1) was first proven by Garsia and Lamperti, [5]. Their work is in the setting of renewal theory so that they assume $\boldsymbol{P}\left\{X_{1} \leqq 0\right\}=0$. However their arguments handle the case when $P\left\{X_{1} \leqq 0\right\} P\left\{X_{1} \geqq 0\right\}>0$. In [5] a lower bound for

$$
\lim _{n \rightarrow \infty} n P(n) G(0, n)
$$

is obtained by extracting an approximating Riemann sum which approaches an integral as $n$ becomes large. This approach is then dropped in favor of a direct attack on

$$
G(0, n)=(1 / 2 \pi) \int_{-\pi}^{\pi} e^{-i n t}(1-\varphi(t))^{-1} d t .
$$

We have used the approximating sum technique of [5] and estimated the error terms which naturally arise. We have not tried to extend to $d>1$ those methods which were finally used in [5] to get their result. The case $d=1$ is cared for here with the same methods which are used to handle $d>1$.

Also in the case $d=1$ and $P\left\{X_{1} \leqq 0\right\}=0$, (1.1) can be obtained for $0<\alpha<1$ as a corollary to a theorem of deBruijn and Erdos, Theorem 3 of [1]. However their result is obtained under the additional assumption: $P(0, n+1) P(0, n-1)-P^{2}(0, n)>0$ for all $n \geqq 2$. Under a monotone condition on $P(0, x)$, one less stringent than this, (1.1) is proven in our Corollary $3-\mathrm{A}$ for $0<\alpha<1$. Corollary 3-B contains results for $d \geqq 2$ and $0<\alpha<2$.

Spitzer in P26.1, [16], obtained (1.2) in the case $\alpha=2, d=3$, $E\left(\left|X_{1}\right|^{2}\right)<\infty$, and $E\left(X_{1}\right)=0$ with $P(|x|)$ replaced by $1 /|x|^{2}$. In the case when $E\left(X_{1}\right) \neq 0$ and when $P(0, x)$ goes to 0 exponentially fast as $|x| \rightarrow \infty$, Ney and Spitzer, [11], have determined explicitly the asymptotic behavior of the relativized Green function $G(x, y) / G(0, y)$, $x$ fixed, for arbitrary $d$. Using their result they obtain a "concrete 
realization" of the Martin boundary for this class of Markov chains. In $\S 4$ there are found some potential theoretic corollaries to the results of this paper.

When the mean is not 0 , Doney, in [2], has determined the asymptotic behavior of $G(x, y)$ in the direction of the mean vector under the assumption that there are [d/2] moments when $d>5$ and $\max (2,[(d+1) / 2])$ moments when $2 \leqq d<5$.

Section 2 contains lemmas that determine which terms can be ignored in the sum which defines $|x|{ }^{d} P(|x|) G(0, x)$. Following the lead of [5] strong use is made of the Karamata Theorem which gives the form of a function of slow growth. Specifically, if $L(x)$ is a measurable function of a real variable satisfying

$$
\lim _{x \rightarrow \infty} \frac{L(k x)}{L(x)}=1
$$

for every $k>0$, then by [8],

(1.3) $L(x)=C(x) \exp \left\{\int_{0}^{x}[\varepsilon(t) / t] d t\right\}$ where $C(x)$ has a finite nonzero limit as $x \rightarrow \infty$ and $\lim _{t \rightarrow \infty} \varepsilon(t)=0$.

Also in $§ 2$, and later in $\S 3$, we need the Lévy Formula, [10], for the characteristic function of a $d$-dimensional stable law, $G_{\alpha}(x), 0<\alpha \leqq 2$.

$$
\int e^{i t \cdot x} d G_{\alpha}(x)=\exp \left\{i A \cdot t-\Lambda|t|^{\alpha}\left[C_{1}(t /|t|)+i C_{2}^{(\alpha)}(t)\right]\right.
$$

where $A$ is a constant vector, $\Lambda$ is a positive constant,

$$
\begin{array}{rlr}
C_{1}(t /|t|) & =\int|\theta \cdot t /| t||^{\alpha} d H(\theta), & \\
C_{2}^{(\alpha)}(t) & =C_{2}^{(\alpha)}(t /|t|)=-\int \operatorname{sgn}(\theta \cdot t) \tan (\pi \alpha / 2)|\theta \cdot t /| t||^{\alpha} d H(\theta) \\
C_{2}^{(1)}(t) & =(2 / \pi) \int(\theta \cdot t /|t|) \ln |\theta \cdot t| d H(\theta), & \text { for } \alpha \neq 1,
\end{array}
$$

and $H$ is a probability measure on the surface of the $d$-dimensional unit sphere. When $d=1$ the above takes the form, [6],

$$
\int e^{i t x} d G_{\alpha}(x)=\exp \left\{i \gamma t-\Lambda|t|^{\alpha}[1+i \beta(t /|t|) \omega(t, \alpha)]\right.
$$

where $\gamma, \Lambda$, and $\beta$ are real constants, $|\beta| \leqq 1, \omega(t, \alpha)=\tan (\pi \alpha / 2)$ for $\alpha \neq 1$, and $\omega(t, 1)=(2 / \pi) \ln |t|$.

Section 5 contains examples which show that (1.1) does not hold for $\alpha \leqq d / 2$ unless additional conditions are imposed on $P(0, x)$, and 
which also show that the behavior of $g_{\alpha}(x)$ for large $x$ can also cause trouble when $\alpha \leqq(d-1) / 2$.

\section{Negligible terms.}

DeFinition. Let $P$ be a real nonincreasing function of a real variable. The function $P^{-1}$ is defined by $P^{-1}(y)=\inf \{x: P(x)<y\}$.

Lemma 2-A. Let $X$ be a random vector with $P(x)=\boldsymbol{P}\{|X|>x\}>0$ for all $x>0$. If for any $k>0$

$$
\lim _{x \rightarrow \infty} P(x) / P(k x)=k^{\alpha},
$$

then for any $k>0$

$$
\lim _{x \rightarrow 0} P^{-1}(x) / P^{-1}(k x)=k^{1 / \alpha}
$$

Proof. The right continuity of $P$ together with (2.1) gives for arbitrary $\varepsilon>0$

$$
\begin{aligned}
(1-\varepsilon)^{\alpha} & =\lim _{y \rightarrow 0} \frac{P\left(P^{-1}(y)\right)}{P\left(P^{-1}(y)[1-\varepsilon]\right)} \leqq \lim _{y \rightarrow 0} \frac{P\left(P^{-1}(y)\right)}{P\left(P^{-1}(y)-1\right)} \\
& \leqq \lim _{y \rightarrow 0} P\left(P^{-1}(y)\right) / y \leqq 1 \text { and hence } \lim _{y \rightarrow 0} P\left(P^{-1}(y)\right) / y=1 .
\end{aligned}
$$

To establish (2.2) assume that there exists a sequence $\left\{y_{n}\right\}$ with $y_{n}>0$ for all $n$ and $\lim _{n \rightarrow \infty} y_{n}=0$, and $\gamma>0$ such that

$$
\varlimsup_{n \rightarrow \infty} P^{-1}\left(y_{n}\right) / P^{-1}\left(k y_{n}\right) \geqq(k+\gamma)^{1 / \alpha} .
$$

Then

$$
\begin{aligned}
1 / k & =\lim _{n \rightarrow \infty} P\left(P^{-1}\left(y_{n}\right)\right) / P\left(P^{-1}\left(k y_{n}\right)\right) \\
& \leqq \varlimsup_{n \rightarrow \infty} P\left(P^{-1}\left(y_{n}\right)\right) / P\left(P^{-1}\left(y_{n}\right)(k+\gamma)^{-1 / \alpha}\right)=1 /(k+\gamma) .
\end{aligned}
$$

But $k \geqq k+\gamma$ is impossible so that

$$
\varlimsup_{y \rightarrow 0} P^{-1}(y) / P^{-1}(k y) \leqq k^{1 / \alpha} .
$$

A similar argument shows that

$$
\lim _{y \rightarrow 0} P^{-1}(y) / P^{-1}(k y) \geqq k^{1 / \alpha}
$$

and hence the lemma is proven.

LEMmA 2-B. Let $\left\{S_{n}, n \in N\right\}$ be a strongly aperiodic d-dimensional 
random walk having increments $X_{1}, X_{2}, X_{3}, \cdots$ belonging to the domain of attraction of a nondegenerate d-dimensional stable law, $G_{\alpha}(x)$, with characteristic exponent $\alpha, 0<\alpha<\min (d, 2)$. If $\boldsymbol{P}\{|x|>x\}=$ $P(x)$ then

$$
\lim _{R \rightarrow \infty}|x|^{d} P(|x|) \sum_{n>R \mid P(|x|)} P_{n}(0, x)=0 \text { uniformly in } x .
$$

Proof. Denote by $\{B(n)\}$ that sequence of constants such that for suitably chosen vectors, $\{A(n)\}, \boldsymbol{P}\left\{S_{n}<B(n)(A(n)+x)\right\}$ converges to $G_{\alpha}(x)$ for each $x=\left(x_{1}, x_{2}, \cdots x_{d}\right)$. From the proof of Theorem 4.2, [14], it can be seen that $B(n)$ can be taken to be $P^{-1}(1 / n)$.

It will be shown first that

$$
\lim _{R \rightarrow \infty}|x|{ }^{d} P(|x|) \sum_{n>R / P(|x|)}(B(n))=0 \text { uniformly in } x .
$$

To this end we can assume $H=1$ and, because $P^{-1}$ is monotone, it is enough to show

$$
\lim _{R \rightarrow \infty}|x|^{d} P(|x|) \int_{R / P(|x|)}^{\infty}\left(P^{-1}(1 / y)\right)^{-d} d y=0 \text { uniformly in } x .
$$

Arguing as in (2.3) we get

$$
\lim _{x \rightarrow \infty} P^{-1}(P(x)) / x=1 .
$$

This fact together with the change of variable, $v=P(|x|) y$, reduces the proof of (2.6) to the establishment of

$$
\lim _{R \rightarrow \infty} \int_{R}^{\infty}\left[\frac{P^{-1}(P(|x|))}{P^{-1}(P(|x|) / y)}\right]^{d} d y=0 \text { uniformly in } x .
$$

Let $J(v)=P^{-1}(1 / v) / v^{1 / \alpha}$. By Lemma 2-A, $\lim _{v \rightarrow \infty} J(v) / J(k v)=1$ for every $k>0 . \quad J$ is then a function of slow growth and hence $J$ has the form

$$
J(v)=C(v) \exp \left\{\int_{0}^{v}[\varepsilon(t) / t] d t\right\}
$$

where $C(v)$ and $\varepsilon(t)$ have the properties stated in (1.3). Now choose $\varepsilon>0$ so that $d / \alpha-d \varepsilon>1$. For $|x|$ so large that $|\varepsilon(t)| \leqq \varepsilon$ and

$$
\frac{C(1 / P(|x|))}{C(y / P(|x|))} \leqq 2
$$

whenever $t>1 / P(|x|)$ and $y>1$, we have

$$
\left[\frac{P^{-1}(P(|x|))}{P^{-1}(P(|x|) / y)}\right]^{d} \leqq\left[\frac{J(1 / P(|x|))}{J(y / P(|x|)) y^{1 / \alpha}}\right]^{d} \leqq 2 y^{-d(1 / \alpha-\varepsilon)} .
$$


The limit in (2.8) is obviously uniform over any finite $x$ set and hence we have the desired uniformity for all $x$.

We return to (2.4). The strongly aperiodic condition means that for any $\varepsilon>0$ there exists $\rho(\varepsilon)<1$ such that for all

$$
t \in C-\{t:|t| \leqq \varepsilon\},|\varphi(t)| \leqq \rho(\varepsilon) .
$$

We write

$$
\begin{aligned}
\left|P_{n}(0, x)\right| & \leqq\left|(2 \pi)^{-d} \int_{C} e^{-i t x} \varphi^{n}(t) d t\right| \\
& \leqq(2 \pi)^{-d}\left[(2 \pi)^{d} \rho^{n}(\varepsilon)+\int_{|t| \leqq \varepsilon}|\varphi(t)|^{n} d t\right]
\end{aligned}
$$

and remark that showing that for fixed $\varepsilon>0$,

$$
\lim _{R \rightarrow \infty}|x|^{d} P(|x|) \sum_{n>R / P(|x|)} \int_{|t| \leqq \varepsilon}|\varphi(t)|^{n} d t=0 \text { uniformly in } x,
$$

proves the lemma. To accomplish this we modify an argument found in the proof of Theorem 6.1 of [14]. Choose $\varepsilon>0$ so small that $|t| \leqq \varepsilon$ implies

$$
\left|2^{\alpha}-\frac{\ln |\varphi(t)|}{\ln |\varphi(t / 2)|}\right|<n<\left(2^{\alpha}-1\right) / 2 .
$$

This is possible by Theorem 4.3 of [14]. Then

$$
\begin{aligned}
& \int_{|t| \leqq \&}|\varphi(t)| d t^{n}=(B(N))^{-d} \int_{|t| \leqq \& B(n)} \mid \varphi\left(t /\left.B(n)\right|^{n} d t\right. \\
& \quad \leqq(B(n))^{-d}\left[\int_{|t| \leqq 1}|\varphi(t / B(n))|^{n} d t+\sum_{m=0}^{k} \int_{2^{m}<|t| \leqq 2^{m+1}}|\varphi(t / B(n))|^{n} d t\right] .
\end{aligned}
$$

Here $k=k(n)$ is chosen such that $2^{k}<\varepsilon B(n) \leqq 2^{k+1}$. But

$$
\begin{gathered}
\sum_{m=0}^{k} \int_{2^{m}<|t| \leqq 2^{m+1}}|\varphi(t / B(n))|^{n} d t \leqq \sum_{m=0}^{k} 2^{m d} \int_{1<|t| \leqq 2}\left|\varphi\left(2^{m} t / B(n)\right)\right|^{n} d t \\
\leqq \sum_{m=0}^{k} 2^{m d} \int_{1<|t| \leqq 2} \exp \left\{n\left(2^{\alpha}-\eta\right)^{m} \ln |\varphi(t / B(n))|\right\} d t
\end{gathered}
$$

and this together with the fact that

$$
\lim _{n \rightarrow \infty} n \ln |\varphi(t / B(n))|=-|t|{ }^{\alpha} C_{1}(t /|t|)
$$

uniformly for $1 \leqq|t| \leqq 2$, the fact that $C_{1}(t /|t|)$ is bounded away from 0 because $G_{\alpha}(x)$ is nondegenerate, and the fact that $2^{\alpha}-\eta>1+\eta$ yields

$$
\int_{|t| \leqq \delta}|\varphi(t)|^{n} d t \leqq(B(n))^{-d} M
$$


where $M$ is a constant independent of $n$. An appeal to (2.5) completes the proof.

LEMma 2-C. Let $\left\{S_{n}, n \in N\right\}$ be a strongly aperiodic d-dimensional random walk having increments $X_{1}, X_{2}, X_{3} \ldots$ belonging to the domain of attraction of a nondegenerate d-dimensional stable law, $G_{\alpha}(x)$, with characteristic exponent $\alpha, d / 2<\alpha<\min (d, 2)$. If $E\left(X_{1}\right)=0$ where it exists then

$$
\lim _{\varepsilon \rightarrow 0}|x|^{d} P(|x|) \sum_{n<\varepsilon \mid P(|x|)} P_{n}(0, x)=0 \text { uniformly in } x .
$$

Here $P(x)=P\left\{\left|X_{1}\right|>x\right\}$.

Proof. Case 1. $d=1$. First we observe that

$$
\begin{aligned}
& |\varphi(t+\pi / x)-\varphi(t)| \leqq \int_{|y|>|x|} 2 d \boldsymbol{P}\left\{X_{1}<y\right\} \\
& \quad+\int_{|y| \leqq|x|}(\pi|y| /|x|) d \boldsymbol{P}\left\{X_{1}<y\right\} \leqq 2 P(|x|)+(\pi /|x|) \int_{0}^{|x|} P(y) d y .
\end{aligned}
$$

If it can be shown that

$$
\lim _{|x| \rightarrow \infty}(1 /|x|) \int_{0}^{|x|}(P(y) / P(|x|)) d y=\int_{0}^{1} d y / y^{\alpha}
$$

then it will be possible to conclude that there exists a positive constant $M$ independent of both $t$ and $x$ such that for all $x, x \neq 0$, and for all $t \in[-\pi, \pi]$,

$$
|\varphi(t+\pi / x)-\varphi(t)| \leqq M P(|x|) .
$$

To establish (2.10) let $L(x)=P(x) x^{\alpha}$. By Theorem 4.2 of [14], $\lim _{x \rightarrow \infty} L(k x) / L(x)=1$ for every $k>0$ and hence $L$ is a function of slow growth. Therefore

$$
L(x)=C(x) \exp \left\{\int_{0}^{x}[\varepsilon(t) / t] d t\right\}
$$

where $C(x)$ and $\varepsilon(t)$ have the properties stated in (1.3). Now choose $\gamma>0$ so that $\alpha+\gamma<1$. For $|x|$ so large that $|\varepsilon(t)| \leqq \gamma$ and $|C(y|x|) / C(|x|)-1| \leqq \gamma$ whenever $t>\gamma|x| P(|x|)$ and $y>\gamma P(|x|)$ we have

$$
\begin{aligned}
(1 /|x|) \int_{0}^{|x|}( & P(y) / P(|x|)) d y=\int_{0}^{1}(P(y|x|) / P(|x|)) d y \\
& \leqq \gamma+\int_{\gamma P(|x|)}^{1}(P(y|x|) / P(|x|)) d y \leqq \gamma+(1+\gamma) \int_{0}^{1} d y / y^{\alpha+\gamma}
\end{aligned}
$$

and also 


$$
(1 /|x|) \int_{0}^{|x|}(P(y) / P(|x|)) d y \geqq \int_{\gamma}^{1} d y / y^{\alpha-r} .
$$

$\gamma$ can be made as small as we like. This proves (2.10), and hence (2.11). (2.11) is stated and proved as Lemma 3.32 in [5] under the additional assumption that $\boldsymbol{P}\left\{X_{1} \leqq 0\right\}=0$. The above proof is the same as that in [5] and is given here only for completeness and to familiarize the reader with arguments involving functions of slow growth. We now use (2.11) to get (2.9) when $d=1$. For $x>0$ and $x$ an integer,

$$
\begin{aligned}
& \int_{-\pi}^{\pi} e^{-i t x} \varphi^{k}(t) d t=\frac{1}{2}\left[\int_{0}^{\pi} e^{i v x}\left(\varphi^{k}(v)-\varphi^{k}(v+\pi / x)\right) d v\right. \\
& \left.\quad+\int_{\pi-\pi / x}^{\pi} e^{-i v x} \varphi^{k}(v+\pi / x) d v-\int_{-\pi / x}^{0} e^{-i v x} \varphi^{k}(v+\pi / x) d v\right] \\
& \quad+\frac{1}{2}\left[\int_{-\pi}^{0} e^{-i v x}\left(\varphi^{k}(v)-\varphi^{k}(v-\pi / x)\right) d v\right. \\
& \left.\quad+\int_{-\pi}^{-\pi+\pi / x} e^{-i v x} \varphi^{k}(v-\pi / x) d v-\int_{0}^{\pi / x} e^{-i v x} \varphi^{k}(v-\pi / x) d v\right] .
\end{aligned}
$$

The above results from letting $t=v+\pi / x$ when $t>0$ and $t=v-\pi / x$ when $t<0$. From (2.12) for $x>0$,

$$
\begin{aligned}
& x P(x) \sum_{n<\varepsilon / P(x)} P_{n}(0, x) \leqq x P(x) \sum_{n=1}^{N} P_{n}(0, x) \\
& \quad+\sum_{n=N}^{[\varepsilon / P(x)]}(x P(x) / 4 \pi)\left[\int_{0}^{\pi}\left|\varphi^{n}(t)-\varphi^{n}(t+\pi / x)\right| d t\right. \\
& \left.\quad+\int_{-\pi}^{0}\left|\varphi^{n}(t)-\varphi^{n}(t-\pi / x)\right| d t\right]+(x P(x) / 4 \pi)(4 \pi / x)(\varepsilon / P(x))
\end{aligned}
$$

where $N$ will be fixed later. For any fixed $j, S_{j}$ belongs to the domain of attraction of a stable law with characteristic exponent $\alpha$. Hence $\boldsymbol{P}\left\{\left|S_{j}\right|>x\right\}=L_{j}(x) / x^{\alpha}$ where $L_{j}$ is a function of slow growth as $x \rightarrow \infty, \quad \alpha>1 / 2$ and therefore the first term on the right in (2.13) is small for large $x$. The last term on the right in (2.13) is a constant times $\varepsilon$. We will show that the second term on the right in (2.13) can be made small for large $x$ and small $\varepsilon$ in a way much like that used to prove Lemma 2-B

$$
\begin{aligned}
& \sum_{n=N}^{[\varepsilon / P(x)]} x P(x)\left[\int_{0}^{\pi}\left|\varphi^{n}(t)-\varphi^{n}(t+\pi / x)\right| d t\right. \\
& \left.+\int_{-\pi}^{0}\left|\varphi^{n}(t)-\varphi^{n}(t-\pi / x)\right| d t\right] \\
& \leqq 2 \sum_{n=N}^{[\varepsilon / P(x)]} x P(x) \int_{0}^{\pi}|\varphi(t)-\varphi(t+\pi / x)| \sum_{k=0}^{n-1}\left|\varphi^{k}(t)\right| \\
& \times\left|\varphi^{n-1-k}(t+\pi / x)\right| d t \leqq 2 M I^{\prime}+2 M I^{\prime \prime}
\end{aligned}
$$


where

$$
I^{\prime}=\sum_{n=N}^{[\varepsilon / P(x)]} x P^{2}(x) \int_{\delta}^{\pi} \sum_{k=0}^{n-1}\left|\varphi^{k}(t)\right|\left|\varphi^{n-1-k}(t+\pi / x)\right| d t
$$

and

$$
\begin{aligned}
I I^{\prime}= & \sum_{n=N}^{[\epsilon / P(x)]} x P^{2}(x) \int_{0}^{\delta B(n)}(B(n))^{-1} \sum_{k=0}^{n-1}\left|\varphi^{k}(t / B(n))\right| \\
& \times \mid \varphi^{n-1-k}((t+B(n) \pi / x) \mid / B(n)) .
\end{aligned}
$$

Here $B(n)=P^{-1}(H / n), \delta$ is chosen so that $0<t<2 \delta$ implies

$$
\left|2^{\alpha}-\frac{\ln |\varphi(t)|}{\ln |\varphi(t / 2)|}\right|<\eta<\left(2^{\alpha}-1\right) / 2,
$$

and $N$ is taken large enough and $\varepsilon$ small enough to insure that $B(n) \pi / x<\delta$ and $n l n|\varphi(v / B(n))| \leqq-C|v|^{\alpha}$ for some $C>0$ uniformly in $v$ for $1 \leqq v \leqq 2+S$ whenever $N \leqq n \leqq \varepsilon / P(x)$. Strong aperiodicity together with $1 / 2<\alpha$ gives $\lim _{x \rightarrow \infty} I^{\prime}=0$. An argument like that used in Lemma $2-\mathrm{B}$ yields

$$
\sum_{k=0}^{n-1} \int_{0}^{\delta B(n)}\left|\varphi^{k}(t / B(n))\right|\left|\varphi^{n-1-k}((t+B(n) \pi / x) / B(n))\right| d t \leqq n c^{\prime}
$$

where $c^{\prime}$ is some positive constant. $x^{\nu}$ times the exponential part of a function of slow growth is eventually monotone for each $\nu, \nu \neq 0$. Hence it is sufficient to replace $I^{\prime \prime}$ by an integral and show it has the desired behavior.

$$
\begin{gathered}
\lim _{x \rightarrow \infty} x P^{2}(x) \int_{N}^{\varepsilon / P(x)}\left[y\left(P^{-1}(1 / y)\right)^{-1}\right] d y=\lim _{x \rightarrow \infty} \int_{N P(x)}^{\varepsilon} y \frac{P^{-1}(P(x)) d y}{P^{-1}(P(x) / y)} \\
\quad=\lim _{x \rightarrow \infty} \int_{N P(x)}^{\varepsilon} \frac{y^{1-1 / \alpha} J(1 / P(x))}{J(y / P(x))} d y=\int_{0}^{\varepsilon} y^{1-1 / \alpha} d y .
\end{gathered}
$$

Passing to the limit under the integral can be justified by exploiting the fact that $J$ is a function of slow growth. Therefore given any $\gamma>0$ there exist $\varepsilon>0$ and $x_{0}>0$ such that

$$
x P(x) \sum_{n<\varepsilon \backslash P(x)} P_{n}(0, x)<\gamma
$$

whenever $\varepsilon<\varepsilon_{0}$ and $x \geqq x_{0}$. The limit in (2.9) is obviously uniform in $x$ for $0 \leqq x \leqq x_{0}$. The estimates for negative $x$ are handled as above except in (2.12) the substitution required is $t=v+\pi / x$ when $t<0$ and $t=v-\pi / x$ when $t>0$.

Case 2. $d=2,3$. Let $t=\left(t_{1}, \cdots, t_{d}\right)$. For $\alpha>1$ if $E\left(X_{1}\right)=0$ then there exists a constant $M>0$ such that for all $t$, 


$$
\left|\frac{\partial \varphi(t)}{\partial t_{i}}\right| \leqq M P(1 /|t|)\left|t^{-1}\right|
$$

To establish (2.15) we write

$$
\begin{aligned}
\left|\frac{\partial \varphi(t)}{\partial t_{i}}\right|= & \mid \int_{\left|X_{1}\right| \leqq 1 /|t|}\left(e^{i t X_{1}}-1\right) X_{1 i} \boldsymbol{P}\{d \omega\} \\
& +\int_{\left|X_{1}\right|>1 /|t|}\left(e^{i t X_{1}}-1\right) X_{1 i} \boldsymbol{P}\{d \omega\} \\
\leqq & \int_{\left|X_{1}\right| \leqq 1 /|t|}\left|X_{1}\right|^{2}|t| \boldsymbol{P}\{d \omega\}+\int_{\left|X_{1}\right|>1 /|t|} 2\left|X_{1}\right| \boldsymbol{P}\{d \omega\} \\
\leqq & \int_{0}^{1 /|t|} 2 y|t| P(y) d y+2 P(1 /|t|)|t|^{-1} \\
& +\int_{1 /|t|}^{\infty} 2 P(y) d y=P(1 /|t|)|t|^{-1} \\
& \times\left[\int_{0}^{1}(2 y P(y /|t|) / P(1 /|t|)) d y\right. \\
& \left.+2+2 \int_{1}^{\infty}(P(y /|t|) / P(1 /|t|)) d y\right]
\end{aligned}
$$

where $X_{1}=\left(X_{11}, \cdots, X_{1 d}\right)$. An argument involving functions of slow growth allows us to let $|t| \rightarrow 0$ under the integral in the right side of (2.16) and this gives the desired result.

The following estimates are also required and can easily be shown to be true.

There exists $M^{\prime}>0$ such that for all $t$ and real $r$

$$
\begin{array}{r}
|t|^{-1}\left|\int_{\left|X_{1}\right| \leqq r}\left(e^{i t X_{1}}-1\right) X_{1 i} P\{d \omega\}\right| \leqq M^{\prime} r^{2} P(r) ; \\
\left|\int_{\left|X_{1}\right|>r}\left(e^{i t X_{1}}-1\right) X_{1 i} P\{d \omega\}\right| \leqq M^{\prime} r P(r) ;
\end{array}
$$

and

$$
\left|\int_{\left|X_{1}\right| \leqq r}\left(e^{i t X_{1}}-1\right) X_{1 i}^{2} P\{d \omega\}\right| \leqq M^{\prime} r^{2} P(r)
$$

Let

$$
Q(j)=\left\{x=\left(x_{1}, x_{2}, \cdots, x_{d}\right) \in R:|x| \leqq\left|x_{j}\right| \sqrt{\bar{d}}\right\} .
$$

We will show that (2.9) holds uniformly in $x$ for $x \in Q(j)$ and since $R \subset \bigcup_{j=1}^{d} Q(j)$, this will prove the lemma. Integrating by parts and applying (2.15), (2.17), (2.18), and (2.19) gives 
$(2.20)$

$$
\begin{aligned}
& \left|\int e^{-i t x} \varphi^{n}(t) d t\right|=\mid \int\left[\left(-i x_{j}\right)^{-1} e^{-i t x} n \varphi^{n-1}(t)\right. \\
& \quad \times\left(\int_{\left|X_{1}\right| \leqq\left|x_{j}\right|} i\left(e^{i t X_{1}}-1\right) X_{1 j} P\{d \omega\}\right. \\
& \left.\left.\quad+\int_{\left|X_{1}\right|>\left|x_{j}\right|} i\left(e^{i t X_{1}}-1\right) X_{1 j} P\{d \omega\}\right)\right] d t \mid \\
& \leqq \\
& \quad \mid \int\left[x _ { j } ^ { - 2 } e ^ { - i t x } n \left(\varphi^{n-1}(t)(-1) \int_{\left|X_{1}\right| \leqq\left|x_{j}\right|}\left(e^{i t X_{1}}-1\right) X_{1 j}^{2} P\{d \omega\}\right.\right. \\
& \left.\left.\quad+(n-1) \varphi^{n-2}(t) \frac{\partial \varphi(t)}{\partial t_{j}} \int_{\left|X_{1}\right| \leqq\left|x_{j}\right|} i\left(e^{i t X_{1}}-1\right) X_{1 j} P\{d \omega\}\right)\right] d t \mid \\
& \quad+M^{\prime} P\left(\left|x_{j}\right|\right) \int n\left|\varphi^{n-1}(t)\right| d t \leqq E^{\prime}+E^{\prime \prime}
\end{aligned}
$$

where

$$
E^{\prime}=2 M^{\prime} P\left(\left|x_{j}\right|\right) \int n\left|\varphi^{n-1}(t)\right| d t
$$

and

$$
E^{\prime \prime}=P\left(\left|x_{j}\right|\right) M M^{\prime} n(n-1) \int\left|\varphi^{n-2}(t)\right| P(1 /|t|) d t .
$$

Again using the fact that $P(y) y^{\alpha}$ is a function of slow growth we get that there exists $M^{\prime \prime}>0$ such that for all

$$
x \in Q(j), P\left(\left|x_{j}\right|\right) \leqq M^{\prime \prime} P(|x|),
$$

also for

$$
|t| \leqq 1, P(B(n) /|t|) \leqq P(B(n))
$$

and $\lim _{n \rightarrow \infty} n P(B(n))=1$. Hence

$$
\begin{aligned}
& |x|^{d} P(|x|) \sum_{n<\varepsilon / P(|x|)} P_{n}(0, x) \leqq|x|^{d} P(|x|)\left[\sum_{n=1}^{N} P_{n}(0, x)\right. \\
& \left.\quad+\sum_{n=N}^{[\varepsilon / P(|x|)]}\left(E^{\prime}+E^{\prime \prime}\right)\right] \leqq|x|{ }^{d} P(|x|) \sum_{n=1}^{N} P_{n}(0, x) \\
& \quad+|x|^{d} P^{2}(|x|)\left[\sum _ { n = N } ^ { [ \varepsilon | P ( | x | ) ] } \int _ { t \in C - \{ t : | t | \leqslant \delta \} } \left[2 M^{\prime} M^{\prime \prime} n\left|\varphi^{n-1}(t)\right|\right.\right. \\
& \left.\quad+M M^{\prime} M^{\prime \prime}(n-1)\left|\varphi^{n-2}(t)\right|\right] d t+\sum_{n=N}^{[\varepsilon / P(|x|)]} n(B(n))^{-d \mid \alpha} \\
& \quad \times \int_{|t| \leqq \delta B(n)}\left[2 M^{\prime} M^{\prime \prime}\left|\varphi^{n-1}(t / B(n))\right|\right. \\
& \left.\left.\quad+2 M M^{\prime} M^{\prime \prime}\left|\varphi^{n-2}(t / B(n))\right|\right] d t\right]
\end{aligned}
$$

where $\delta$ is small. Arguments like those found in the case where $d=1$ complete the proof. The critical term following the passage 
to the limit in $|x|$ is $\int_{0}^{\varepsilon} y^{1-d / \alpha} d y$. For $\alpha>d / 2, y^{1-d / \alpha}$ is integrable on $[0, \varepsilon]$ and hence (2.9) holds.

3. Proof of the theorem. We first prove the following lemma.

LemmA 3-A. Let $g_{\alpha}(\Lambda, x)$ be a probability density function on $E^{d}$ satisfying

$$
\int e^{i t x} g_{\alpha}(\Lambda, x) d x=\exp \left\{-\Lambda|t|^{\alpha}\left[C_{1}(t /|t|)+i C_{2}^{(\alpha)}(t)\right]\right\}
$$

where $C_{1}(t /|t|)$ and $C_{2}^{(\alpha)}(t)$ are defined in (1.4). Then there exists a constant $M=M(\Lambda)$ such that for all $x$,

$$
g_{\alpha}(\Lambda, x) \leqq M /|x| \text {. }
$$

Proof.

$$
\begin{aligned}
g_{\alpha}(\Lambda, x) & =\int g_{\alpha}(\Lambda / 2, x-y) g_{\alpha}(\Lambda / 2, y) d y \\
& =\int_{|y| \leqq|x| / 2} g_{\alpha}(\Lambda / 2, x-y) g_{\alpha}(\Lambda / 2, y) \\
& +\int_{|y|>|x| / 2} g_{\alpha}(\Lambda / 2, x-y) g_{\alpha}(\Lambda / 2, y) d y \\
& \leqq 2 M^{\prime} \sum_{i=1}^{d} \boldsymbol{P}\left\{\left|Y_{i}\right|>|x| / 2 \sqrt{d}\right\}
\end{aligned}
$$

where $M^{\prime}$ is a uniform upper bound for $g_{\alpha}(\Lambda / 2, x)$ and $Y=\left(Y_{1}, Y_{2}, \cdots Y_{d}\right)$ is a random vector with density $g_{\alpha}(\Lambda / 2, x)$. Each $Y_{i}$ has a one dimensional distribution function which is stable with characteristic exponent $\alpha$ and hence by [6], p. 182, I, $|x|{ }^{\alpha} \boldsymbol{P}\left\{\left|Y_{i}\right|>|x|\right\}$ is asymptotically constant for each $i$. This proves the lemma.

Proof of the theorem. Initially we assume that the random walk is strongly aperiodic. Under this additional assumption we first show that the limit in (1.1) holds for fixed direction $u_{x}=x /|x|$. Let $\delta>0$ be given. By Lemmas 2-B, 2-C, and 3-A, $\varepsilon$ can be chosen so small and $R$ so large that

$$
\begin{gathered}
|x|^{d} P(|x|) \sum_{n<\varepsilon / P(|x|)} P_{n}(0, x)<\delta,|x|^{d} P(|x|) \sum_{n>R \mid P(|x|)} P_{n}(0, x)<\delta, \\
\alpha \int_{0}^{R-1 / \alpha} g_{\alpha}\left(v u_{x}\right) v^{d-1-\alpha} d v=\int_{R}^{\infty} g_{\alpha}\left(v^{-1 / \alpha} u_{x}\right) v^{-d / \alpha} d v<\delta
\end{gathered}
$$

and

$$
\int_{\varepsilon-1 / \alpha}^{\infty} g_{\alpha}\left(v u_{x}\right) v^{d-1-\alpha} d v=\int_{0}^{\varepsilon} g_{\alpha}\left(v^{-1 / \alpha} u_{x}\right) v^{-d / \alpha} d v<\delta
$$


The local limit theorem for $d$-dimensional lattice random vectors, Theorem 6.1, [14], allows us to write

$$
P_{n}(0, x)=(B(n))^{-d}\left[G_{\alpha}(x / B(n))+e(n, x)\right]
$$

where $\lim _{n \rightarrow \infty} e(n, x)=0$ uniformly in $x$. Also

$$
\begin{aligned}
& \lim _{|x| \rightarrow \infty}(n P(|x|))^{1 / \alpha}|x| / B(n) \\
& =\lim _{|x| \rightarrow \infty} P^{-1}(P(|x|)) n^{1 / \alpha} /(P(|x|))^{-1 / \alpha} P^{-1}(1 / n) \\
& =\lim _{|x| \rightarrow \infty} \frac{J(1 / P(|x|))}{J(n)}=1 \text { uniformly in } n \text { for } \\
& \quad[\varepsilon / P(|x|)] \leqq n \leqq[R / P(|x|)] .
\end{aligned}
$$

Hence

$$
\begin{aligned}
& \lim _{|x| \rightarrow \infty}|x|^{d} P(|x|) \sum_{n=[\varepsilon / P(|x|)]}^{[R / P(|x|)]}(B(n))^{-d} g_{\alpha}\left(|x| u_{x} / B(n)\right) \\
& \quad=\lim _{|x| \rightarrow \infty} \sum_{n=[\varepsilon / P(|x|)]}^{[R / P(|x|)]} g_{\alpha}\left(u_{x}(n P(|x|))^{-1 / \alpha}\right)(n P(|x|))^{-d / \alpha} P(|x|) \\
& =\int_{\varepsilon}^{R} g_{\alpha}\left(v^{-1 / \alpha} u_{x}\right) v^{-d / \alpha} d v
\end{aligned}
$$

and

$$
\lim _{|x| \rightarrow \infty}|x|^{d} P(|x|) \sum_{n=[\varepsilon / P(|x|)]}^{[R / P(|x|)]}(B(n))^{-d} e(n, x)=0 .
$$

From (3.3) through (3.7) it is possible to conclude that (1.1) holds for fixed $u_{x}$. The approximating sum technique used in the above argument is due to [5] as was mentioned in $\S 1$.

To show that the limit in (1.1) is actually uniform in $u_{x}$ requires an additional argument. Set

$$
F(r, u)=r^{d} P(r) \sum_{n=[\varepsilon / P(r)]}^{[R / P(r)]}(B(n))^{-d} g_{\alpha}(r u / B(n))
$$

where $r$ is a real number and $|u|=1$. The estimates in (3.3) and (3.7) hold uniformly in $u_{x}$ and hence showing that

$$
\lim _{r \rightarrow \infty} F(r, u)=\int_{\varepsilon}^{R} g_{\alpha}\left(v^{-1 / \alpha} u\right) v^{-d / \alpha} d v=h(u) \text { uniformly in } u
$$

will complete the proof of (1.1). If (3.8) did not hold uniformly in $u$ then there would exist $\varepsilon_{0}>0$ and sequences $\left\{u_{n}\right\}$ and $\left\{r_{n}\right\}, r_{n} \rightarrow \infty$ such that for all $n,\left|F\left(r_{n}, u_{n}\right)-h\left(u_{n}\right)\right|>\varepsilon_{0}$. The unit sphere is compact so that we can assume that there exists $u_{0}$ with $\lim _{n \rightarrow \infty}\left|u_{n}-u_{0}\right|=0$. Then 


$$
\begin{aligned}
\varepsilon_{0} & <\left|F\left(r_{n}, u_{n}\right)-h\left(u_{n}\right)\right| \leqq\left|F\left(r_{n}, u_{n}\right)-F\left(r_{n}, u_{0}\right)\right| \\
& +\left|F\left(r_{n}, u_{0}\right)-h\left(u_{0}\right)\right|+\left|h\left(u_{0}\right)-h\left(u_{n}\right)\right| \text { holds for all } n .
\end{aligned}
$$

The uniform continuity of $g_{\alpha}(x)$ together with the fact that

$$
r^{d} P(r) \sum_{n=[\varepsilon / P(r)]}^{[R / P(r)]}(B(n))^{-d}
$$

is bounded in $r$ gives $\lim _{n \rightarrow \infty}\left|F\left(r_{n}, u_{n}\right)-F\left(r_{n}, u_{0}\right)\right|=0$. Clearly

$$
\lim _{n \rightarrow \infty}\left|F\left(r_{n}, u_{0}\right)-h\left(u_{0}\right)\right|=0 \text { and } \lim _{n \rightarrow \infty}\left|h\left(u_{n}\right)-h\left(u_{0}\right)\right|=0 .
$$

(3.9) is, therefore, impossible. Thus we have (3.8), and hence (1.1) in the case when the random walk is strongly aperiodic. To get (1.1) in the case when the random walk is aperiodic but not strongly aperiodic we make use of a technique found in p. 26.1, [16]. For $0<\nu<1$, let $P^{\prime}(0, x)=\nu P(0, x)$ and $P^{\prime}(0,0)=1-\nu . \quad \sum_{x} P^{\prime}(0, x)=1$ because $P(0,0)=0 . \quad P^{\prime}(0,0)>0$ implies that the random walk defined by $P^{\prime}(0, x)$ is strongly aperiodic. Also $G(0, x)=\nu G^{\prime}(0, x), P^{\prime}(|x|)=$ $\nu P(|x|), 1-\varphi^{\prime}(t)=\nu(1-\varphi(t))$, and

$$
\ln \left(\int e^{i t x} g_{\alpha}^{\prime}(x) d x\right)=\nu\left(\ln \left(\int e^{i t x} g_{\alpha}(x) d x\right)\right) .
$$

Here the prime indicates that the expression is defined in terms of $P^{\prime}(0, x)$. Form the above it also follows that

$$
\nu \int_{0}^{\infty} g_{\alpha}^{\prime}\left(v u_{x}\right) v^{d-1-\alpha} d v=\int_{0}^{\infty} g_{\alpha}\left(v u_{x}\right) v^{d-1-\alpha} d v .
$$

Hence $|x|{ }^{d} P(|x|) G(0, x)=|x|{ }^{d} P^{\prime}(|x|) G^{\prime}(0, x)$ and this expression approaches $(\alpha / \nu) \int_{0}^{\infty} g_{\alpha}\left(v u_{x}\right) v^{d-1-\alpha} d v$ as $|x| \rightarrow \infty$ uniformly in $u_{x}$. $\nu$ can be taken arbitrarily close to 1 and therefore the proof of (1.1) is complete.

(1.2) follows immediately. If $\alpha>1$ then by Theorem 1, [17], $g_{\alpha}(x)>0$ for all $x$. In the case $d=1$ since we are substracting no sequence of constants from $S_{n} / B(n)$,

$$
\gamma=\lim _{\varepsilon \rightarrow 0^{+}} \lim _{n \rightarrow \infty}(B(n))^{-1} \sum_{k=1}^{n} \int_{|x| \leqq \& B(n)} x d \boldsymbol{P}\left\{X_{k}<x\right\}=0 .
$$

When $\gamma=0$ a necessary and sufficient condition for there to be positive probability on both $(0, \infty)$ and $(-\infty, 0)$ is $|\beta|<1$, [15]. In each of the above cases $\alpha \int_{0}^{\infty} g_{\alpha}\left(v u_{x}\right) v^{d-1-\alpha} d v$ is bounded away from 0 and that is enough to give (1.2).

In the corollaries dealing with $\alpha \leqq d / 2$ we require in addition that:

$$
\text { There exists } K>0 \text { such that for all } x \in R
$$$$
|x|{ }^{d} P(0, x) / P(|x|) \leqq K \text {. }
$$ 
CoRollary 3-A. Let $\left\{S_{n}, n \in N\right\}$ be an aperiodic one-dimensional random walk having increments belonging to the domain of attraction of a nondegenerate stable low with density $g_{\alpha}(x)$. If $(3.10)$ is satisfied then (1.1) holds for all $\alpha$ satisfying $1 / 4<\alpha<1$. If we assume that

$$
\begin{aligned}
& \text { There exists } \rho>0 \text { such that if }|x| \geqq \rho \text { then } \\
& P(0, x) \geqq P(0, x+x /|x|)
\end{aligned}
$$

then (1.1) holds for all $\alpha$ satisfying $0<\alpha<1$.

Proof. We need only establish that (2.9) holds and in doing so we may assume that the random walk is strongly aperiodic.

First we show that under (3.10) with $1 / 4<\alpha<1$, (2.13) can be estimated in such a way as to yield (2.9). Applying to

$$
\int_{0}^{\pi}\left|\varphi^{n}(t)-\varphi^{n}(t+\pi / x)\right| d t
$$

first Hölder's inequality with $2>q>1 / 2 \alpha$ and then the HausdorffYoung inequality, [18], gives

$$
\begin{aligned}
\int_{0}^{\pi}\left|\varphi^{n}(t)-\varphi^{n}(t+\pi / x)\right| d t \leqq & \left(\int_{-\pi}^{\pi}|\varphi(t)-\varphi(t+\pi / x)|^{P} d t\right)^{1 / P} \\
& \times\left(\int_{0}^{\pi}\left|\sum_{k=0}^{n-1} \varphi^{n-1-k}(t) \varphi^{k}(t+\pi / x)\right|^{q} d t\right)^{1 / q} \\
\leqq & \left(\sum_{k=-\infty}^{\infty}\left(K \sqrt{2 \pi} P(|k|)\left|1-e^{i \pi k / x}\right| /|k|\right)^{q}\right)^{1 / q} \bar{\Gamma} n(B(n))^{-1 / q} \\
\leqq & \Gamma x^{-1+1 / q} n P(x)(B(n))^{-1 / q}
\end{aligned}
$$

where $\Gamma$ and $\bar{\Gamma}$ are positive constants independent of $x$ and $n$. A similar estimate can be made on the integral over $[-\pi, 0]$ in $(2.13)$. These bounds applied to (2.13) give (2.9) and hence (1.1) for $1 / 4<\alpha<1$.

Under (3.11) $P(|x|) \geqq|x|(P(0,2 x)+P(0,-2 x))$ for all large $|x|$ and hence (3.11) implies (3.10). By taking $d=1$ in the proof of Corollary 3 -B it can be seen that (3.11) gives $P_{n}(0, x) \leqq c|x|^{-1}$ for all $n \geqq 1$ and all $x, x \neq 0$. Here $c$ is some constant. From this estimate, (2.9), and hence (1.1), follows.

Under the additional assumption that $g_{\alpha}(x)$ places positive probability on both sides of 0 , it is possible to conclude (1.2) in the above corollary.

The fact that for $1 / 4<\alpha<1 / 2$ (3.10) implies (1.1) is noted in [5]. We have modified their technique to get our result for $1 / 4<\alpha \leqq 1 / 2$.

For any sequence $a(x), x=\left(x_{1}, x_{2}, \cdots, x_{d}\right)$, denote by $a\left(x ; b_{k_{1}}, b_{k_{2}}, \cdots, b_{k_{n}}\right)$ the sequence $a(x)$ with $x_{k_{j}}$ fixed and equal to $b_{k_{j}}$ for $j=1,2, \cdots, n$. We define the difference operator, $D_{k}$, by $D_{k}(a(x))=a\left(x ; x_{k}\right)-a\left(x ; x_{k}+1\right)$ if $x_{k} \geqq 0$ and $D_{k}(a(x))=a\left(x ; x_{k}\right)-a\left(x ; x_{k}-1\right)$ if $x_{k}<0$. One of the 
conditions we will impose in higher dimensions, the one needed together with (3.10) to justify $d$ integrations by parts, reduces to (3.11) when $d=1$. Specifically:

$$
\begin{aligned}
& \text { There exists } \rho>0 \text { such that for any } x,|x| \geqq \rho, \\
& D_{1} D_{2} \cdots D_{d}(P(0, x)) \geqq 0 .
\end{aligned}
$$

(3.13) immediately implies that for any $x,|x| \geqq \rho$, and any set of distinct integers, $k_{1}, k_{2}, \cdots, k_{n}, k_{n} \leqq d, D_{k_{1}} D_{k_{2}} \cdots D_{k_{n}}(P(0, x)) \geqq 0$. Also to avoid the difficulty pointed out in Example $5-\mathrm{B}$ we will require that

$$
\lim _{N \rightarrow \infty} \int_{N}^{\infty} g_{\alpha}\left(v u_{x}\right) v^{d-1-\alpha} d v=0 \text { uniformly in } u_{x} .
$$

One method for varifying conditions (3.13) and (3.14) is suggested by the approach found in Example 5-C.

Corollary 3-B. For $d \geqq 2$ let $\left\{S_{n}, n \in N\right\}$ be an aperiodic $d$ dimensional random walk having increments belonging to the domain of attraction of a nondegenerate d-dimensional stable law with density $g_{\alpha}(x)$. Also let (3.10), (3.13), and (3.14) be satisfied. Then:

( I ) (1.1) holds for all $\alpha$ such that $0<\alpha<1$;

(II) (1.2) holds in the case when $\alpha=1$ and the random walk is symmetric;

(III) (1.2) holds in the case when $1<\alpha<2$ and $E\left(X_{1}\right)=0$.

Proof. It is sufficient to prove the corollary for strongly aperiodic random walk. Let $C_{j}=\left\{t \in C: t_{i} \neq 0\right.$ for $\left.1 \leqq i \leqq d, i \neq j\right\}$. It will be shown that for $\nu \leqq d, \partial^{(\nu)} \varphi(t) / \partial t_{j}^{(\nu)}$ exists on $C_{j}$ except when $\nu=d$ and $t_{j}=0$. Also it will be shown that $d$ integrations by parts in the variable $t_{j}$ of $\int_{C_{j}} e^{-i t x} \varphi^{n}(t) d t$ is possible. Let $b\left(t_{j}, \nu\right)=\left|\prod_{k=1}^{\nu} t_{i_{k}}\right|^{-1 / \nu}$ where $j=i_{1}$ and $i_{1}, i_{2}, \cdots, i_{\nu}$ are distinct integers. Finally it will be shown that there exists some $M>0$ which is independent of $t$ such that

$$
\text { For } \nu \geqq \alpha,\left|\frac{\partial^{(\nu)} \varphi(t)}{\partial t_{j}^{(\nu)}}\right| \leqq M\left(b\left(t_{j}, \nu\right)\right)^{\nu} P\left(1 / b\left(t_{j}, \nu\right)\right)
$$

and such that

$$
\text { For } 1<\alpha<2,\left|\frac{\partial \varphi(t)}{\partial t_{j}}\right| \leqq M P(1 /|t|)|t|^{-1} .
$$

When all of the above is accomplished then the usual change of variables, $t_{k}=v_{k} / B(n)$, in each of the terms that arise in the $d$ integrations by parts together with the facts: $\int_{C-C_{j}} d t=0$; the right 
side of (3.15) is integrable in each $t_{i_{k}}$; and $|x|{ }^{\alpha} P(|x|)$ is a function of slow growth; gives $|x|{ }^{d} P_{n}(0, x)$ is bounded uniformly in $n$ and $x$ for $n \geqq 1$ and $x \in Q(j)$. From this, (2.9) follows. (3.14) and (2.9) imply (1.1). As before, Theorem 1 of [17] then gives (1.2) when $1<\alpha<2$. The results of Theorem 1, [17], are not applicable when $\alpha=1$ but the fact that for fixed $u_{x}, g_{1}\left(v u_{x}\right)$ can be extended to a function analytic in an open strip which includes the real $v$ axis means that $g_{1}(x)$ can not be identically 0 on a ray from 0 . This is enough to deduce (1.2) from (1.1) when $\alpha=1$.

We now turn to the proofs of the assertions made above. Let $a(x)$ be a sequence for which (3.13) holds. Let $x$ be in $R$ and let $b_{k_{1}}, b_{k_{2}}, \cdots, b_{k_{m}}$ be integers with the property that when the $x_{k_{i}}$ are replaced by the $b_{k_{i}}, i=1,2, \cdots, m$, then $|x|>\rho$. If $c_{k_{1}}, c_{k_{2}}, \cdots, c_{k_{m}}$ are integers such that $c_{k_{i}}$ and $b_{k_{i}}$ have the same sign and such that $\left|c_{k_{i}}\right|>\left|b_{k_{i}}\right|$ for each $i, i=1,2, \cdots, m$, then

$$
\left|\sum_{x_{k_{i}}=b_{k_{i}}}^{c_{k_{i}}} \ldots \sum_{x_{k}=b_{b_{m}}}^{c_{k_{m}}} e^{i t x} a(x)\right| \leqq 2^{m} a\left(x ; b_{k_{i}}, \cdots, b_{k_{m}}\right)\left|\prod_{j=1}^{m}\left(1-e^{i t_{j}}\right)\right|^{-1} .
$$

The proof of (3.17) proceeds by induction on $m$. We assume that $0<b_{k_{1}}<c_{k_{1}}$. If $c_{k_{1}}<b_{k_{1}}<0$ then appropriate changes in sign must be made in what follows.

$$
\begin{aligned}
& \sum_{x_{k_{1}}=b_{k_{1}}}^{c_{k_{1}}} e^{i t x} a(x)=\sum_{v_{k_{1}}=b_{k_{1}}}^{c_{k_{1}}}\left[\frac{e^{i t_{k_{1}} b_{k_{1}}}-e^{i t_{k_{1}}\left(v_{k_{1}}+1\right)}}{1-e^{i t_{k_{1}}}}\right]\left[a\left(x ; v_{k_{1}}\right)-a\left(x ; v_{k_{1}}+1\right)\right] \\
& +\left[\frac{e^{i t_{k_{1}} b_{k_{1}}}-e^{i t_{k_{1}}\left(c_{k_{1}}+1\right)}}{1-e^{i t_{k_{1}}}}\right]\left[a\left(x ; c_{k_{1}}+1\right)\right] .
\end{aligned}
$$

Taking absolute values and applying (3.13) proves (3.17) for the case $m=1$. To complete the induction all we need do is observe that for fixed $v_{k_{1}}$ the sequences $a\left(x ; v_{k_{1}}\right)-a\left(x ; v_{k_{1}}+1\right)$ and $a\left(x ; c_{k_{1}}+1\right)$ also satisfy (3.13).

Next fix $t \in C_{j}$. Let $C(N)=\left\{x=\left(x_{1}, \cdots, x_{d}\right) \in R:\left|x_{i}\right| \leqq N\right.$ for each $i=1,2, \cdots, d\}$. Applying (3.17) and (3.10) gives

$$
\begin{gathered}
\left|\sum_{x \in C(M)-C(N)} e^{i t x} x_{j}^{\nu} P(0, x)\right| \leqq 2^{d} \sum_{j=N}^{d M} 2^{d-1}\left|\prod_{\substack{k=1 \\
k \neq j}}^{d}\left(1-e^{i t_{k}}\right)\right|^{-1} x_{j}^{\nu} K P\left(x_{j}\right) x_{j}^{-d} \\
+2^{d}(d-1) \sum_{x_{j}=1}^{N} 2^{d-1}\left|\prod_{\substack{k=1 \\
k \neq j}}^{d}\left(1-e^{i t_{k}}\right)\right|^{-1} x_{j}^{\nu} K P\left(x_{j}\right)\left(x_{j}^{2}+N^{2}\right)^{-d / 2}
\end{gathered}
$$

whenever $\rho<N<M$. Hence for fixed $t_{1}, \cdots, t_{j-1}, t_{j+1}, \cdots, t_{d}$ with

$$
\prod_{\substack{i=1 \\ i \neq j}}^{d} t_{i} \neq 0, \sum_{x \in C(N)} e^{i t x} x_{j}^{\nu} P(0, x)
$$


converges uniformly in $t_{j}$ for each $\nu, \nu \leqq d-1$. Therefore on $C_{j}$ for each integer

$$
\nu, \nu \leqq d-1, \frac{\partial^{(\nu)}}{\partial t_{j}^{(\nu)}} \varphi(t)
$$

exists, is continuous in $t_{j}$ for $-\Pi \leqq t_{j} \leqq \Pi$, and

$$
\frac{\partial^{(\nu)}}{\partial t_{j}^{(\nu)}} \varphi(t)=\lim _{N \rightarrow \infty} \sum_{x \in C(N)} e^{i t x}\left(i x_{j}\right)^{\nu} P(0, x) \text {. }
$$

To justify the formula for the $d$ th partial derivative we need to alter slightly the preceeding estimates. Fix $t_{1}, t_{2}, \cdots, t_{d}$ so that $\left|\Pi_{k=1}^{d}\left(1-e^{i t x}\right)\right| \neq 0$. For $\rho<N<M$ we have

$$
\begin{aligned}
& \left|\sum_{\substack{x \in C(M)-C(N) \\
x_{j} \geq 0}} e^{i t x} x_{j}^{d} P(0, x)\right|=N^{d}\left|\sum_{\substack{x \in C(M)-C(N) \\
x_{j} \geqq N}} e^{i t x} P(0, x)\right| \\
& \quad+\left|\sum_{\substack{x \in C(M)-C(N) \\
x_{j} \geq N}} \sum_{k=N d}^{x_{j}^{d}} e^{i t x} P(0, x)\right|+\left|\sum_{\substack{x \in C(M)-C(N) \\
1 \leq x_{j}<N}} \sum_{\substack{k=1 \\
x_{j}}} e^{i t x} P(0, x)\right| \\
& \quad \leqq N^{d} 4^{d} K\left|\prod_{k=1}^{d}\left(1-e^{i t k}\right)\right|^{-1} N^{-d} P(N) \\
& \quad+\sum_{j=N^{d}}^{\infty} 4^{d} K\left|\prod_{k=1}^{d}\left(1-e^{i t k}\right)\right|^{-1}\left(j^{1 / d}\right)^{-d} P\left(j^{1 / d}\right) \\
& \quad+\sum_{j=1}^{N d}(d-1) 4^{d} K\left|\prod_{k=1}^{d}\left(1-e^{i t k}\right)\right|^{-1}\left(j^{2 / d}+N^{2}\right)^{-d / 2} P\left(\left(j^{2 / d}+N^{2}\right)^{1 / 2}\right)
\end{aligned}
$$

as a consequence of (3.10), (3.17) and a change in the order of summation. We can write down a similar expression for the case when $x_{j} \leqq 0$. Therefore if

$$
\prod_{\substack{k=1 \\ k \neq j}}^{d}\left(1-e^{i t k}\right) \neq 0
$$

then $\sum_{x \in C(N)} e^{i t} x_{j}^{d} P(0, x)$ converges uniformly in $t_{j}$ for $0<\varepsilon \leqq\left|t_{j}\right| \leqq \Pi$ and hence we can write

$$
\frac{\partial^{(d)} \varphi(t)}{\partial t_{j}^{(d)}}=\lim _{N \rightarrow \infty} \sum_{x \in C(N)} e^{i t x}\left(i x_{j}\right)^{d} P(0, x) .
$$

In the estimates of the preceeding paragraph replacing $d$ by $\nu$ and $N$ by $b\left(t_{j}, \nu\right)$ and using familiar properties of functions of slow growth gives

(3.18) There exists a constant $\bar{M}>0$ such that

$$
\sup _{N \geq b\left(t_{j}, \nu\right)}\left|\sum_{\left.x \in C^{\prime} N\right)} \sum_{C\left(b\left(t_{j}, \nu\right)\right)} e^{i t x} x_{j}^{\nu} P(0, x)\right| \leqq \bar{M}\left(b\left(t_{j}, \nu\right)\right)^{\nu}\left(P\left(1 / b\left(t_{j}, \nu\right)\right)\right. \text {. }
$$


(3.18) combined with

$$
\left|\sum_{x \in C\left(b\left(t_{j}, \nu\right)\right)} e^{i t x} x_{j}^{\nu} P(0, x)\right| \leqq \sum_{x \in C\left(b\left(t_{j}, \nu\right)\right)} K|x|^{v-d} P(|x|) P(|x|)
$$

gives (3.15) except when $\alpha=1=\nu$. In this special case (3.15) still holds because the symmetry condition of (II) implies that

$$
\sum_{x \in C\left(b\left(t_{j}, 1\right)\right)} \cos (t x) x_{j} P(0, x)=0 .
$$

To get (3.16) subtract $0=\sum_{x \in R} x_{j} P(0, x)$ from $\partial \varphi(t) / \partial t_{j}$ and estimate as in Lemma 3-C by truncating at $|t|^{-1}$. This completes the proof.

If $\sum_{R(j)}|x|^{d} P(0, x)<\infty$, where $R(j)$ is any one of the regions bounded by coordinate planes, and if either $\alpha \neq 1$ or $\alpha=1$ and $B(n)=0(n)$, then condition (3.13) can be removed from $P(0, x)$ for $x \in R(j)$ without affecting either the proofs or the conclusions of the previous corollaries.

In (I) of Corollary 3-B (1.2) may be concluded if it is known that the limit probability measure is not carried on an open half-plane which excludes the origin.

4. Potential theoretic corollaries. The Martin boundary for a transient random walk can be constructed in the following way. Assume $G(\mathbf{0}, y)>0$ for each $y \in R$. Identify each $y \in R$ with the function $G(x, y) / G(0, y)$. A sequence $\left\{y_{n}\right\}, y_{n} \in R$, will be called Cauchy if $\lim _{n \rightarrow \infty}\left|y_{n}\right|=\infty$ and for each $x \in R$ the sequence $\left\{G\left(x, y_{n}\right) / G\left(0, y_{n}\right)\right\}$ is Cauchy with respect to the usual metric on the real line, or if there exist a $y_{0} \in R$ and an integer $n^{\prime}$ such that for all $n \geqq n^{\prime}, y_{n}=y_{0}$. Two Cauchy sequences $\left\{y_{n}\right\}$ and $\left\{v_{n}\right\}$ will be said to belong to the same equivalence class of sequences if either $\lim _{n \rightarrow \infty}\left|y_{n}\right|=\lim _{n \rightarrow \infty}\left|v_{n}\right|=\infty$ and for each $x \in R \lim _{n \rightarrow \infty} G\left(x, y_{n}\right) / G\left(0, y_{n}\right)=\lim _{n \rightarrow \infty} G\left(x, v_{n}\right) / G\left(0, v_{n}\right)$, or $y_{n}=v_{n}=y_{0} \in R$ for all sufficiently large $n$. Complete $R$ in the usual way to get $R^{*}$. Each $\nu \in R^{*}-R=B$ can be identified with a function $F(x), x \in R$, satisfying $\sum_{y \in R} P(x, y) F(y) \leqq F(x)$. Hence we have:

Corollary 4-A. Let $\left\{S_{n}, n \in N\right\}$ be a random walk having the property that (1.2) holds. Then the construction described above gives the one-point compactification of $R$. The point in $B$ is identified with the harmonic function $F(x) \equiv 1, x \in R$.

The extreme harmonic functions for any random walk are always exponentials, [4]. If $E\left(X_{1}\right)=0$ then a convexity argument shows that in the case $P(x, y)=P(0, y-x)$ the only exponential functions satisfying $\sum_{y \in R} P(x, y) h(y)=h(x)$ are the constant functions. Hence if a random walk with $1<\alpha<2$ and $E\left(X_{1}\right)=0$ satisfies those conditions 
given in this paper under which (1.2) holds, then $\sum_{x \in R} P(x, y) h(y)=h(x)$ is impossible unless $h$ is constant. Therefore in this case Corollary 4-A contains as new information only the statement that $B$ contains no points identified with strictly subharmonic functions. A conjecture found in [11] states that if $E\left(X_{1}\right)=0$, then $B$ always consists of a single point. Corollary 4-A provides additional supporting evidence for this conjecture.

For $\alpha<1$ there exists random walks with nonconstant exponential harmonic functions for which (1.1) holds. In the case $d=1$ to impose the condition $|\beta|<1$ is to ask that there be so much probability in the tails of $P(0, x)$ that $\sum_{x \in R} P(0, x) e^{v x}=\infty$ for all $v \neq 0$. Hence here again the corollary yields as a new result only the statement that for no sequence, $\left\{y_{n}\right\}$, can $G\left(x, y_{n}\right) / G\left(0, y_{n}\right)$ have a strictly subharmonic limit.

[3] and [7] contain a complete discussion of the Martin boundary for general Markov chains including theorems on the stochastic convergence of $G\left(x, S_{n}\right) / G\left(0, S_{n}\right)$.

If (1.2) holds and, in addition, if

$$
\lim _{|x| \rightarrow \infty} P(|x|)|x|^{\alpha}=b, 0<b<\infty
$$

then $G(x, y)$ is well enough behaved to permit the use of the Wiener test.

Corollary 4-B. Let $A$ be any infinite set and define $A_{n}$ by $A_{n}=A \cap\left\{x \in R: 2^{-(n+1)} \leqq G(0, x)<2^{-n}\right\}$. Let $C\left(A_{n}\right)$ denote the capacity of $A_{n}$ as defined in D25.3, [16]. If (1.2) and (4.1) hold then $A$ is recurrent if and only if $\sum_{n=1}^{\infty} C\left(A_{n}\right) 2^{-n}=\infty$.

Corollary 4-B is an immediate consequence of [9]. (4.1) will hold if the normalizing constants, $B(n)$, can be taken to be $B n^{1 / \alpha}$ where $B$ is a constant. In this case $G(x, y)$ behaves like $|x-y|^{-(d-\alpha)}$ for large values of $|x-y|$, that is, like the Riesz kernel of order $\alpha$, $\alpha<\min (d, 2)$. [9] also contains a paragraph which describes the classical Wiener test in the theory of Brownian motion.

The following is the discrete version of a theorem of Port's, [12]. The assumptions made are those of our main theorem except we ask only that $0<\alpha<\min (d, 2)$. When $\alpha=1$ we do require that the $A(n)$ can be taken to be 0 .

CoRollary 4-C. Let $B$ be any finite subset of $R$ and set $T_{B}=$ $\min \left\{n: n \geqq 1, S_{n} \in B\right\}$. Then under the above assumptions we have 
(4.2) $\lim _{n \rightarrow \infty}(B(n))^{d} n^{-1} \boldsymbol{P}\left\{n<T_{B}<\infty\right\}=\boldsymbol{P}\left\{T_{B}=\infty\right\} C(B) g_{\alpha}(0)[(d / \alpha)-1]^{-1}$.

Here $C(B)$ is the capacity of $B$.

In the strongly aperiodic case a proof of (4.2) can be obtained by writing out Port's proof, [12], in discrete notation. Techniques found in our work are required only to get

$$
\lim _{n \rightarrow \infty}(B(n))^{d} n^{-1} \sum_{k>n} P_{k}(0, x)=g_{\alpha}(0) \int_{1}^{\infty} y^{-d / \alpha} d y .
$$

The local limit theorem of [13] can be used to prove (4.2) in the case when the random walk is aperiodic but not strongly aperiodic.

The proof and conclusion in corollary 4-C carry over to the case when $d>2=\alpha$ and also to the case when the random walk starts at an arbitrary $x \in R$.

5. Examples, Example 5-A. For $k$ an integer and $k \neq 0$ let

$$
f_{k}=\left\{\begin{array}{l}
c l n|k| /|k|^{1+d / 2}, k \neq 2^{n} \text { for some integer } n . \\
c /|k|^{d / 2} l n|k|, k=2^{n} \text { for some integer } n .
\end{array}\right.
$$

Here $d=1,2$, or 3 and $c$ is chosen such that $\sum_{k} f_{k}=1$. We have

$$
\lim _{m \rightarrow \infty} \frac{m^{d / 2}}{\ln (m)} \sum_{|k|>m} f_{k}=D, 0<D<\infty
$$

so that the distribution function associated with $\left\{f_{k}\right\}$ belongs to the domain of attraction of a stable law with characteristic exponent $d / 2$. The function of slow growth, $x^{d / 2} \sum_{|k|>x} f_{k}$, is asymptotic to $D \ln x$ as $x \rightarrow \infty$. Needed in obtaining (5.1) is the fact that if $E(m)=$ $\left\{k:|k|>m, k=2^{n}\right.$ for some integer $\left.n\right\}$ then

$$
\lim _{m \rightarrow \infty} \frac{m^{d / 2}}{\ln (m)} \sum_{k \in E(m)} f_{k}=0=\lim _{m \rightarrow \infty} \frac{m^{d / 2}}{\ln (m)} \sum_{k \in E(m)} c /|k|^{d / 2} \ln |k| .
$$

Now let $\left\{S_{n}, n \in N\right\}$ be a $d$-dimensional random walk with $S_{k}-S_{k-1}=$ $X_{k}=\left(X_{k 1}, \cdots, X_{k d}\right)$ where the components of $X_{k}$ are also mutually independent and $\boldsymbol{P}\left\{X_{k i}=j\right\}=f_{j}, i=1, \cdots, d$. For this random walk $\overline{\lim }_{|x| \rightarrow \infty}|x|^{d} P(|x|) P(0, x)=v>0$ and hence (1.1) cannot possibly hold. Similar constructions yield examples with $\alpha<d / 2$ where (1.1) does not hold.

EXAMPLE 5-B. Let $\left\{S_{n}, n \in N\right\}$ be a random walk with increments $S_{k}-S_{k-1}=\left(X_{k 1}, \cdots, X_{k d}\right)$ composed of mutually independent symmetric, components each belonging to the domain of attraction of a stable law with characteristic exponent $\alpha, \alpha \leqq(d-1) / 2$ and $\alpha<2$. Along any 
coordinate axis $g_{\alpha}\left(v u_{x}\right) v^{d-1-\alpha} \sim v^{d-2-2 \alpha}$ and hence along any such ray $g_{\alpha}\left(v u_{x}\right)^{d-1-\alpha}$ is not integrable. For such $d$ and $\alpha$, proving (2.9) is therefore not enough to insure (1.1) or (1.2). Required in addition is a bound like that in (3.1) but with a larger exponent on $|x|$.

ExAmple 5-C. Let $\left\{S_{n}, n \in N\right\}$ be a $d$-dimensional random walk with $P(0, x)=b\left(a+|x|^{2}\right)^{-\langle a+\alpha) / 2}$ where $b>0$ and $a>0$. (3.10) obviously holds. Consider $P(0, x)$ as a function defined over all of $E^{(d)}$ by the above formula. $\partial P(0, x) / \partial x_{1} x_{2} \cdots x_{d}$ does not change sign on any region $R(j)$ bounded by coordinate planes. Integrating each of the $x_{k}$ over an interval of length 1 gives $D_{1} D_{2} \cdots D_{d}(P(0, x)) \geqq 0$ and hence (3.13) is satisfied. Next let $A$ and $B$ be spherical neighborhoods on the $d$-dimensional unit sphere such that $A$ and $B$ have the same radius. Set $u_{x}=x /|x|$; then

$$
\lim _{\rho \rightarrow \infty}\left[\sum_{\substack{x i \geq \rho \\ u_{x} \in A}} P(0, x)\right]\left[\sum_{\substack{x \in \geq \rho \\ u_{x} \in B}} P(0, x)\right]^{-1}=1
$$

and hence by Theorem 4.2 of [14] the characteristic function of the associated limit law is $\exp \left\{-\Lambda|T|^{\alpha}\right\}, A>0$. This insures (3.14) and hence Corollary $3-\mathrm{B}$ can be applied to this example.

The above method can also be used to show that Corollary 3-B is applicable when $P(0, x)=\Delta g_{\alpha}(x)$ where $x \in R, \Delta$ is a normalizing constant, and

$$
\int_{E^{(d)}} e^{2 t y} g_{\alpha}(y) d y=\exp \left\{-\Lambda|T|^{\alpha}\right\}, \Lambda>0
$$

I would like to thank F. Spitzer, P. Erodos and H. Kesten for their helpful suggestions and comments.

\section{BIBLIOGRAPHY}

1. N. G. deBruijn and P. Erdos, On a recursion formula and some Tauberian theorems,

J. Res. Nat. Bur. Stand. 50 (1953), 161-164.

2. R. A. Doney, An analogue of the renewal theorem in higher dimensions, Proc. of London Math. Soc., Clarendon Press, Oxford, 1966, 669-684.

3. J. L. Doob, Discrete potential theory and boundaries, J. Math. and Mech. 8 (1959), 433-458.

4. - J. L. Snell and R. E. Williamson, Application of boundary theory to sums of independent random variables, Contributions to probability and statistics, Stanford University Press, Stanford, California, 1960, 182-197.

5. A. Garsia and J. Lamperti, A discrete renewal theorem with infinite mean, Commentarii Mathematici Helvetici 37 (1963), 221-234.

6. B. V. Gnedenko and A. N. Kolmogorov, Limit Distributions for Sums of Independent Random Variables, Addison-Wesley, Cambridge, Mass., 1954.

7. G. A. Hunt, Markov chains and Martin boundaries, Illinois J. Math. 4 (1960), 313340 . 
8. H. J. Karamata, Sur un mode de croissance reguliere, Bull. Soc. Math. de France 61 (1933), 55-62.

9. J. Lamperti, Wiener's test and Markov chains, J. of Math. Analysis and Applications 6 (1963), 58-66.

10. P. Levy, Theorie de l'addition des variables aleatoires, Gauthier-Villars, Paris, 1937.

11. P. Ney and F. Spitzer, The Martin boundary for random walk, Trans. Amer. Math. Soc. 121 (1966), 116-132.

12. S. C. Port, Hitting times for transient stable processes, J. Math. and Mech. 16 (1967), 1229-1246.

13. G. S. Rinehart and J. A. Williamson, Remarks on the periodic behavior in time of sums of random variables (to appear)

14. E. L. Rvaceva, On domains of attraction of multi-dimensional distributions, Selected Translations in Mathematical Statistics and Probability II (1962), 183-205. L'vov. Gos. Univ. Uc. Zap. 29 Ser. Meh.-Mat. 6 (1954), 5-44.

15. A. V. Skorohod, Asymptotic formulas for stable distribution laws, Selected Translations in Mathematical Statistics and Probability I (1961), 157-161. Dokl. Akad. Nauk SSSR. 98 (1954), 731-734.

16. F. Spitzer, Principles of Random Walk, Van Nostrand, Princeton, N. J., 1964.

17. S. J. Taylor, Sample path properties of a transient stable process, Pacific J. Math. 21 (1967), 161-165.

18. A. Zygmund, Trigonometrical Series, Chelsea. 1952.

\section{CORNELL UNIVERSITY}

Received April 28, 1967. This research was partially supported by National Science Foundation Grant GP 4867. 



\section{PACIFIC JOURNAL OF MATHEMATICS}

\section{EDITORS}

\section{H. ROYDEN}

Stanford University

Stanford, California
J. DugundJI

Department of Mathematics

Rice University

Houston, Texas 77001

RICHARD ARENS

University of California

Los Angeles, California 90024

Seattle, Washington 98105

\section{ASSOCIATE EDITORS}
E. F. BeCKENBACH
B. H. NeumanN
F. WOLF
K. YOSIDA

\section{SUPPORTING INSTITUTIONS}

\author{
UNIVERSITY OF BRITISH COLUMBIA \\ CALIFORNIA INSTITUTE OF TECHNOLOGY \\ UNIVERSITY OF CALIFORNIA \\ MONTANA STATE UNIVERSITY \\ UNIVERSITY OF NEVADA \\ NEW MEXICO STATE UNIVERSITY \\ OREGON STATE UNIVERSITY \\ UNIVERSITY OF OREGON \\ OSAKA UNIVERSITY \\ UNIVERSITY OF SOUTHERN CALIFORNIA
}

\author{
STANFORD UNIVERSITY \\ UNIVERSITY OF TOKYO \\ UNIVERSITY OF UTAH \\ WASHINGTON STATE UNIVERSITY \\ UNIVERSITY OF WASHINGTON \\ * * * * \\ AMERICAN MATHEMATICAL SOCIETY \\ CHEVRON RESEARCH CORPORATION \\ TRW SYSTEMS \\ NAVAL WEAPONS CENTER
}

Mathematical papers intended for publication in the Pacific Journal of Mathematics should be in typed form or offset-reproduced, double spaced with large margins. Underline Greek letters in red, German in green, and script in blue. The first paragraph or two must be capable of being used separately as a synopsis of the entire paper. It should not contain references to the bibliography. Manuscripts, in duplicate if possible, may be sent to any one of the four editors. All other communications to the editors should be addressed to the managing editor, Richard Arens, University of California, Los Angeles, California 90024.

Each author of each article receives 50 reprints free of charge; additional copies may be obtained at cost in multiples of 50 .

The Pacific Journal of Mathematics is published monthly. Effective with Volume 16 the price per volume (3 numbers) is $\$ 8.00$; single issues, $\$ 3.00$. Special price for current issues to individual faculty members of supporting institutions and to individual members of the American Mathematical Society: $\$ 4.00$ per volume; single issues $\$ 1.50$. Back numbers are available.

Subscriptions, orders for back numbers, and changes of address should be sent to Pacific Journal of Mathematics, 103 Highland Boulevard, Berkeley 8, California.

Printed at Kokusai Bunken Insatsusha (International Academic Printing Co., Ltd.), 7-17, Fujimi 2-chome, Chiyoda-ku, Tokyo, Japan.

PUBLISHED BY PACIFIC JOURNAL OF MATHEMATICS, A NON-PROFIT CORPORATION

The Supporting Institutions listed above contribute to the cost of publication of this Journal, but they are not owners of publishers and have no responsibility for its content or policies. 


\section{Pacific Journal of Mathematics \\ Vol. 25, No. $2 \quad$ October, 1968}

Martin Aigner, On the tetrahedral graph ..................... 219

Gregory Frank Bachelis, Homomorphisms of annihilator Banach

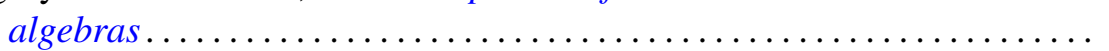

Phillip Alan Griffith, Transitive and fully transitive primary abelian groups.......................................... 249

Benjamin Rigler Halpern, Fixed points for iterates . . . . . . . . . . . . . 255

James Edgar Keesling, Mappings and dimension in general metric spaces ......................................... 277

$\mathrm{Al}$ (Allen Frederick) Kelley, Jr., Invariance for linear systems of ordinary differential equations ................................ 289

Hayri Korezlioglu, Reproducing kernels in separable Hilbert spaces . . . . . 305

Gerson Louis Levin and Wolmer Vasconcelos, Homological dimensions and Macaulay rings ................................. 315

Leo Sario and Mitsuru Nakai, Point norms in the construction of harmonic

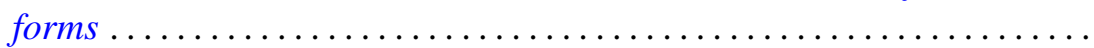

Barbara Osofsky, Noncommutative rings whose cyclic modules have cyclic injective hulls ..................................... 331

Newton Tenney Peck, Extreme points and dimension theory........... 341

Jack Segal, Quasi dimension type. II. Types in 1-dimensional spaces ...... 353

Michael Schilder, Expected values of functionals with respect to the Ito distribution ...

Grigorios Tsagas, A Riemannian space with strictly positive sectional curvature

John Alexander Williamson, Random walks and Riesz kernels . . 